\title{
Arrhythmogenic Right Ventricular Cardiomyopathy in a 20-year-old Woman with Systemic Sclerosis
}

\author{
Kaoru Dohi ${ }^{1}$, Shiro Nakamori ${ }^{1}$, Tairo Kurita ${ }^{1}$, Youichi Omoto ${ }^{2}$, Masaki Ishida ${ }^{3}$, \\ Mashio Nakamura ${ }^{4}$, Kyoko Imanaka-Yoshida ${ }^{5}$, Hajime Sakuma ${ }^{3}$, \\ Hitoshi Mizutani ${ }^{2}$ and Masaaki Ito ${ }^{1}$
}

\begin{abstract}
A 20-year-old Japanese woman with systemic sclerosis was evaluated for the progressive aggravation of chest pain, palpitations and dyspnea. Cardiac magnetic resonance imaging revealed diffuse wall thinning, segmental dyskinesis and late gadolinium enhancement of the right ventricular (RV) myocardium. Cardiac catheterization demonstrated no pulmonary hypertension. 24-hour electrocardiography monitoring showed nonsustained ventricular tachycardia (VT). Pulseless VT was induced via programmed ventricular stimulation. An endomyocardial biopsy of the right side of the interventricular septum was performed, the histological specimen of which demonstrated massive myocardial atrophy and fibro-fatty replacement with predominant fibrotic changes. The patient was ultimately diagnosed with arrhythmogenic RV cardiomyopathy.
\end{abstract}

Key words: systemic sclerosis, arrhythmogenic right ventricular cardiomyopathy, sudden death

(Intern Med 53: 973-977, 2014)

(DOI: 10.2169/internalmedicine.53.1977)

\section{Introduction}

Systemic sclerosis (SSc) is a systemic disease characterized by fibrosis, vascular damage and autoimmunity that affects many organs. We herein report the case of a young Japanese woman with SSc whose clinical course was malignant due to the presence of arrhythmogenic right ventricular cardiomyopathy (ARVC) associated with sudden cardiac death. A literature search identified only 10 cases of SSc with ARVC in Japan, including the present case. This case involved the youngest patient, in whom the right ventricular (RV) myocardium was severely damaged.

\section{Case Report}

A 20-year-old Japanese woman with an 11-year history of SSc was admitted to our hospital due to the chronic and progressive aggravation of chest pain, palpitations and dyspnea. The patient had noticed Raynaud's phenomenon and was diagnosed with SSc at 9 years of age based on the presence of sclerotic changes extending from the fingers to the forearms with positive serum anti-Scl70 antibodies. She was initially treated with oral prednisolone. However, the skin sclerosis gradually extended to the face and trunk, and she was ultimately diagnosed with diffuse type SSc. At 15 years of age, she experienced her first episode of palpitations with chest pain lasting for less than 10 seconds. 24-hour electrocardiography (ECG) monitoring showed 1,400 monofocal ventricular contractions, including a few couplets. A treadmill exercise test showed a negative result for ischemic changes, and transthoracic echocardiography demonstrated a normal cardiac function without structural abnormalities. Although the patient's symptoms were self-limited and stable for three years, her condition progressively worsened after 18 years of age. Thereafter, she was repeatedly admitted to

\footnotetext{
${ }^{1}$ Department of Cardiology and Nephrology, Mie University Graduate School of Medicine, Japan, ${ }^{2}$ Department of Dermatology, Mie University Graduate School of Medicine, Japan, ${ }^{3}$ Department of Radiology, Mie University Graduate School of Medicine, Japan, ${ }^{4}$ Department of Clinical Cardiovascular Research, Mie University Graduate School of Medicine, Japan and ${ }^{5}$ Department of Pathology and Matrix Biology, Mie University Graduate School of Medicine, Japan

Received for publication October 23, 2013; Accepted for publication December 5, 2013
}

Correspondence to Dr. Kaoru Dohi, dohik@clin.medic.mie-u.ac.jp 


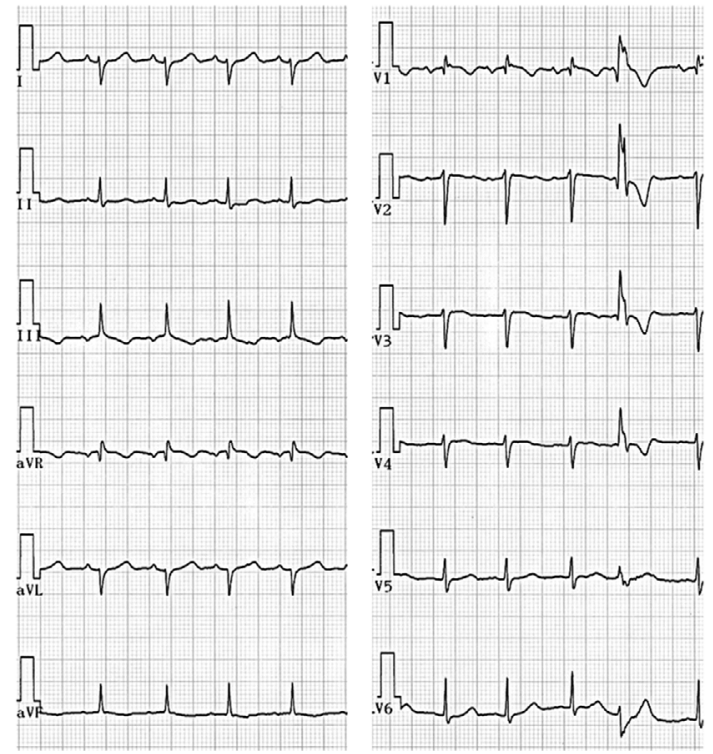

Figure 1. A 12-lead electrocardiography showed right axis deviation of the QRS complex, incomplete right bundle branch block and negative $T$ waves in the III, $\mathrm{V}_{\mathrm{F}}$ and $\mathrm{V}_{1}$ leads with ventricular premature contractions.

the dermatology department with ulceration of the fingers and toes. She ultimately suffered from more severe chest pain and exertional dyspnea and was referred to our cardiology unit at 20 years of age. Blood tests revealed a plasma brain natriuretic peptide level of $103 \mathrm{pg} / \mathrm{mL}$ and a troponin$\mathrm{T}$ level of $0.04 \mathrm{ng} / \mathrm{mL}$. A 12-lead ECG showed right axis deviation of the QRS complex, incomplete right bundle branch block and negative $\mathrm{T}$ waves in the III, ${ }_{\mathrm{a}} \mathrm{V}_{\mathrm{F}}$ and $\mathrm{V}_{1}$ leads with ventricular premature contractions (Fig. 1). A chest X-ray showed a mildly enlarged cardiac silhouette with a cardiothoracic ratio of $53 \%$. Computed tomography (CT) revealed moderate bilateral interstitial fibrosis. 24-hour ECG monitoring showed non-sustained ventricular tachycardia (VT). Two-dimensional echocardiography demonstrated a severely impaired RV function with chamber dilatation associated with leftward shift of the interventricular septum. The RV fractional area change was only $20 \%$, as assessed on the apical four-chamber view. Trace tricuspid regurgitation was observed, with an estimated maximal RV-right atrial pressure gradient during systole of $23 \mathrm{mmHg}$. Cine magnetic resonance imaging (MRI) revealed diffuse wall thinning, segmental dyskinesis and a small right ventricular aneurysm at the junction of the RV lateral wall with the diaphragmatic wall. The RV end-diastolic volume index, endsystolic volume index and ejection fraction determined on cine MRI were $158 \mathrm{~mL} / \mathrm{m}^{2}, 127 \mathrm{~mL} / \mathrm{m}^{2}$ and $19 \%$, respectively (Fig. 2A and B). Late gadolinium-enhanced MRI (LGE-MRI) showed that the RV myocardium throughout the free wall and apical portion of the interventricular septum, as well as the basal interventricular septum, had been replaced with fibrous tissue (Fig. 2C). Noncontrast-enhanced CT demonstrated that a portion of the RV free wall had been replaced with adipose tissue (Fig. 2D). Cardiac cathe- terization disclosed angiographically normal coronary arteries without pulmonary arterial hypertension (PAH) (mean pulmonary arterial pressure: $15 \mathrm{mmHg}$ and pulmonary vascular resistance index: 215 dyne $\times \sec \times \mathrm{cm}^{-5} \times \mathrm{m}^{-2}$ ). An endomyocardial biopsy of the right side of the interventricular septum was performed, the histological specimen of which revealed massive myocardial atrophy and fibro-fatty replacement with predominant fibrotic changes. Residual myocytes were distinctly less than $50 \%$ according to a visual estimation (Fig. 3). Pulseless VT with a cycle length of $201 \mathrm{msec}$ was induced via programmed ventricular stimulation with triple extrastimuli at the RV apex. The QRS morphology included a left bundle block with a superior axis configuration, suggesting that the VT had originated from the base of the right ventricular inferior wall (Fig. 4). The patient met three major task force criteria and was ultimately diagnosed with ARVC (1). She received an implantable cardioverter defibrillator for the primary prevention of sudden cardiac death. However, she suddenly died two years later in her home. An autopsy was not performed.

\section{Discussion}

We experienced the case of a young woman with SSc and ARVC. The heart is a major organ involved in SSc, with an estimated clinical prevalence of cardiac involvement of 15 $35 \%(2,3)$. Although cardiac manifestations remain subclinical in the majority of patients with SSc, individuals who develop clinically apparent myocardial manifestations are recognized to be at greater risk of clinical deterioration, and monitoring myocardial involvement represents an important aspect of disease management (4). Cardiac manifestations of SSc can affect all structures of the heart and may result in pericardial effusion, arrhythmias, conduction system defects, valvular impairment, myocardial ischemia, myocardial hypertrophy and heart failure. PAH is a frequent and wellknown complication of SSc. Therefore, it has been recognized that patients with SSc develop RV structural and functional abnormalities secondary to RV pressure overload. Therefore, primary RV involvement has rarely been reported and may be underestimated in patients with SSc. Hachulla et al. examined 52 consecutive patients with SSc $(56 \pm 11$ years old) and found that abnormal findings on cardiac MRI were not uncommon in the patients with limited type $(n=32)$ or diffuse type $(n=20)$ SSc (5). There were seven patients with RV dilatation and three patients with RV segmental wall motion abnormalities in whom PAH was excluded based on the results of catheterization. The authors speculated that RV structural and functional abnormalities are not specific for PAH and may correspond to the findings of specific cardiac involvement in SSc patients. However, the causal mechanisms underlying RV abnormalities were not specified in that study, and there were no patients with late gadolinium enhancement on LGE-MRI or increased signal intensity on T2-weighted MRI in the RV segments.

In the present case, the patient exhibited no evidence or 

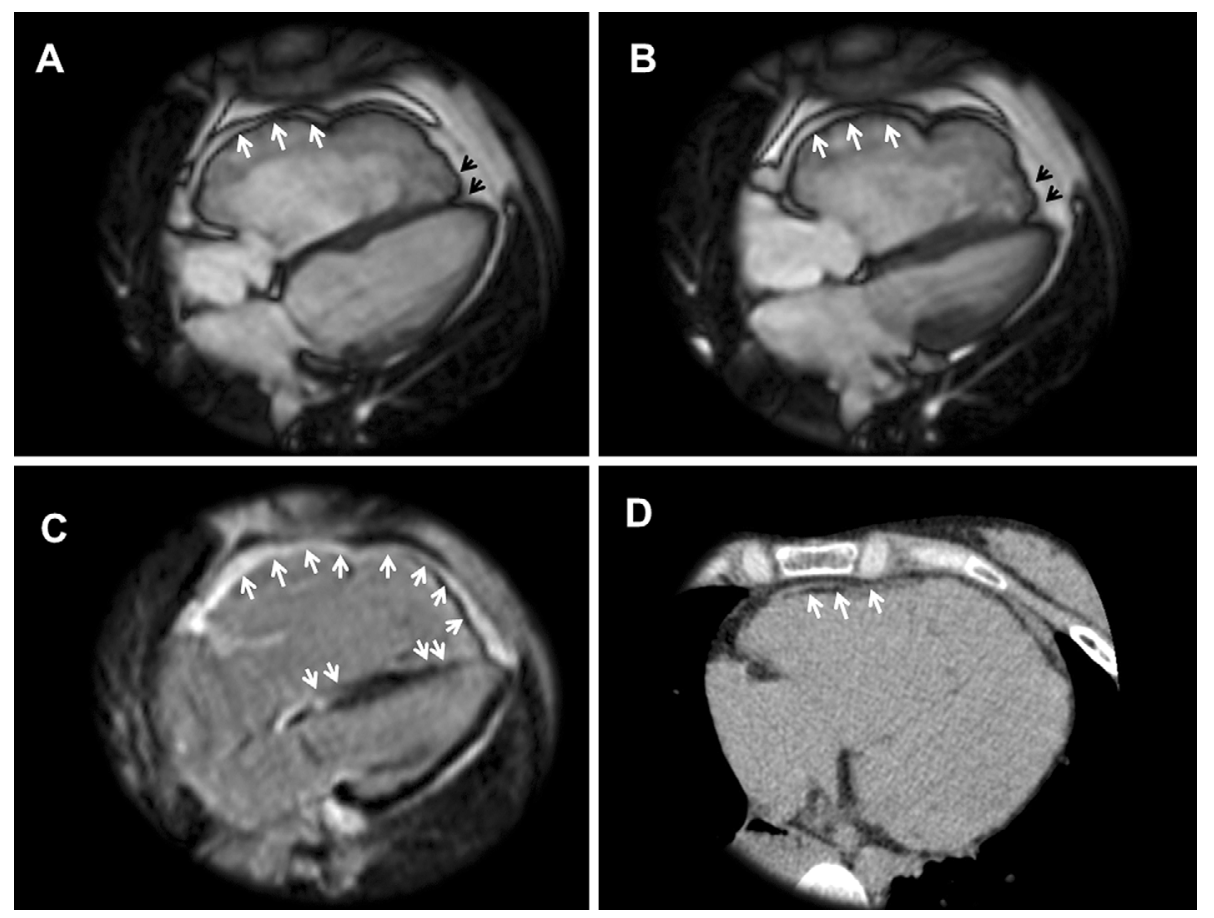

Figure 2. Cine MRI revealed diffuse wall thinning, segmental dyskinesis (white arrows) and a small right ventricular aneurysm at the junction of the $\mathrm{RV}$ lateral wall with the diaphragmatic wall (black arrows, Panel A: end-diastole, and Panel B: end-systole). Late gadolinium-enhanced MRI showed late gadolinium enhancement of the RV myocardium throughout the free wall and apical portion of the interventricular septum, as well as the basal interventricular septum (Panel C, white arrows), while noncontrast-enhanced CT demonstrated that a portion of the RV free wall had been replaced with adipose tissue (Panel D, white arrows).
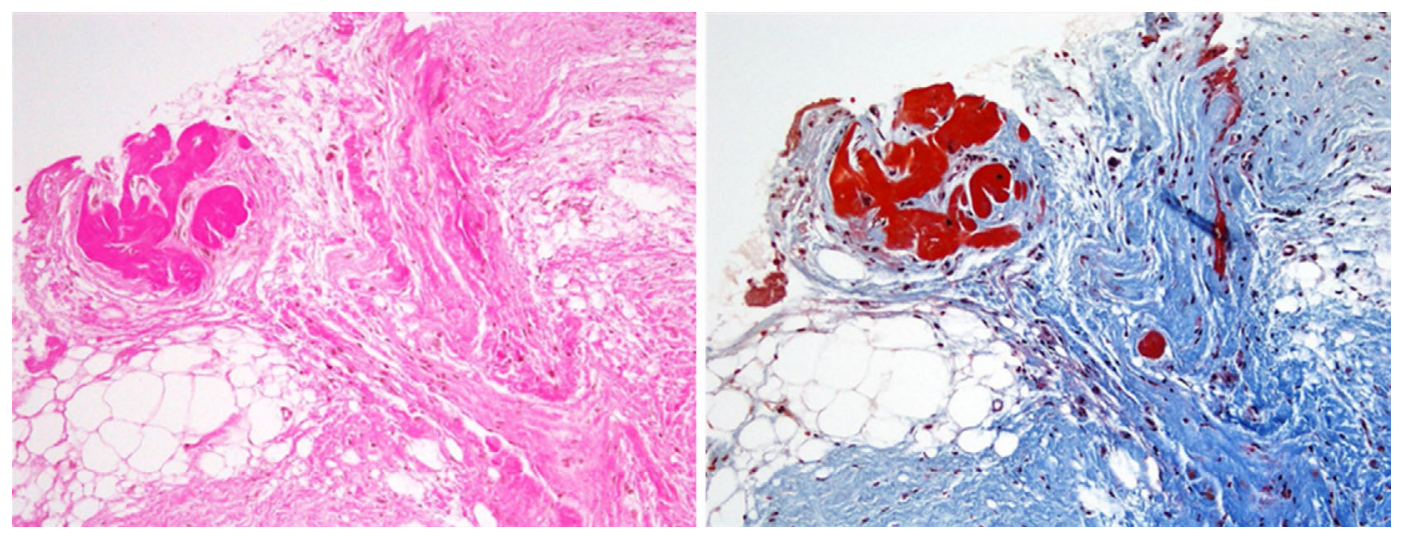

Figure 3. The histological specimens demonstrated massive myocardial atrophy and fibro-fatty replacement with predominant fibrotic changes (Left: Hematoxylin and Eosin staining sections, and right: Masson Trichrome-stained sections).

signs of PAH. Therefore, her RV structural and functional abnormalities primarily resulted from RV myocardial insult. Cardiac MRI showed severe RV chamber dilatation, wall motion abnormalities and a large extent of late gadolinium enhancement in the RV myocardium, especially in the free wall, indicating myocardial fibrosis. The histopathological examination revealed that most of the cardiomyocytes had been replaced with fibro-fatty tissue. The QRS morphology of the VT included left bundle block with a superior axis configuration. Therefore, the patient was ultimately diag- nosed with ARVC based on the task force criteria (1). ARVC is a RV cardiomyopathy characterized by an abnormal RV morphology and ventricular arrhythmias that can cause sudden cardiac death. Replacement of the myocardium with fibro-fatty tissue is a characteristic feature of $\operatorname{ARVC}(6,7)$. ARVC is associated with a highly variable clinical course and broad spectrum of symptoms and electri$\mathrm{cal} / \mathrm{structural}$ abnormalities. The present patient's symptoms were presumed to result from her cardiac disease, as both conditions became progressively aggravated simultaneously. 


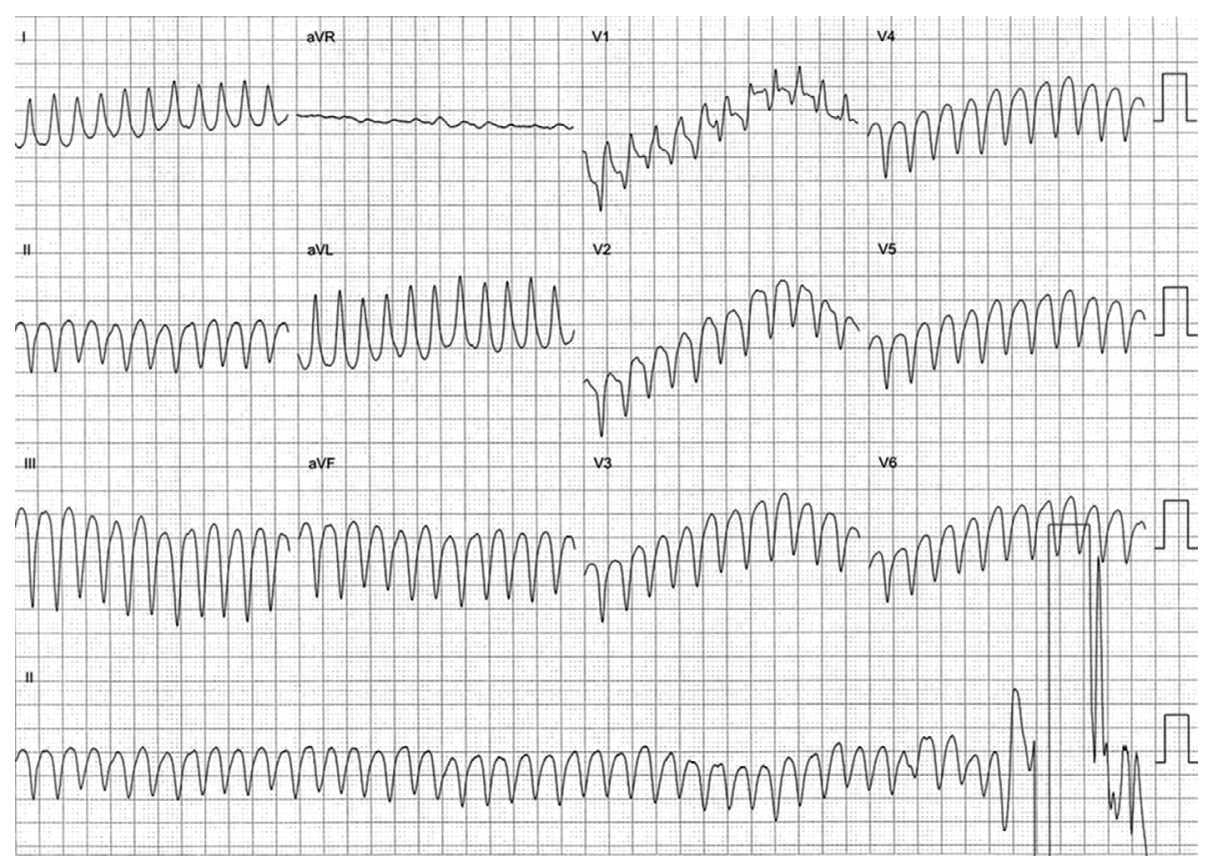

Figure 4. Ventricular tachycardia with a cycle length of 201 msec induced via programmed ventricular stimulation with triple extrastimuli at the right ventricular apex.

A retrospective review of the patient's chart demonstrated a normal cardiac function without structural abnormalities on the transthoracic echocardiography images recorded at 15 years of age. Only 10 cases of ARVC associated with SSc have been reported in Japan, including the present case (8). Therefore, we speculate that RV involvement is not rare in the setting of SSc, although primary and isolated RV involvement, including ARVC, remains uncommon. In patients with ARVC, genetic variations have been found in desmosomes (1), major cell adhesion junctions that are particularly prominent in the epidermis and cardiac tissue and important for the rigidity and strength of cells. Seven genes associated with ARVC have been identified: plakoglobin, desmoplakin, plakophilin-2, desmoglein-2, desmocollin-2, transforming growth factor beta-3 and TMEM43 (1). The intracellular regions of desmosomal cadherins, such as desmoglein-2, interact with plakoglobin and plakophilin, which in turn link to desmoplakin and consequently with the intermediate filament network. Due to the particularly important roles of desmosomes in the heart and skin, desmosomal protein mutations can produce specific cardio/cutaneous phenotypes. Recent studies have demonstrated that impairment of actin assembly as a result of desmoglein-2/integrin-beta8 complex formation is a major factor contributing to angiogenesis deregulation in patients with SSc (9). Accordingly, some forms of RV involvement in SSc patients are associated with desmosomal malfunctions that induce intercellular disruption and angiogenesis deregulation in the right ventricle.

Cardiac MRI has been reported to detect fibro-fatty changes associated with ARVC with high sensitivity. These findings differ from the fibrotic myocardial changes commonly found in SSc patients on MRI $(5,10)$. In the present case, cardiac MRI was very useful for detecting fibrous tis- sue replacement in the myocardium and correctly diagnosed ARVC. Fat replacement of the RV without fibrosis likely represents a distinct clinicopathological entity that is not synonymous with ARVC. The detection of fibrous tissue replacement in the myocardium is more suggestive of arrhythmogenic than fat replacement. In the present case, fibrous tissue replacement of the myocardium was much more prominent than fatty tissue replacement on the histological examination, consistent with the MRI findings.

In conclusion, the present case is rare and involves the youngest known patient with ARVC complicated by SSc. Although the prevalence of primary and isolated RV myocardial involvement in patients with SSc is very low, this finding is an important characteristic of organ involvement in the setting of SSc. This case report reinforces the need to deeply investigate all patients for global cardiac involvement.

The authors state that they have no Conflict of Interest (COI).

\section{References}

1. Marcus FI, McKenna WJ, Sherrill D, et al. Diagnosis of arrhythmogenic right ventricular cardiomyopathy/dysplasia: proposed modification of the task force criteria. Circulation 121: 1533-1541, 2010.

2. Ferri C, Valentini G, Cozzi F, et al. Systemic sclerosis: demographic, clinical, and serologic features and survival in 1,012 Italian patients. Medicine 81: 139-153, 2002.

3. Steen VD, Medsger TA Jr. Severe organ involvement in systemic sclerosis with diffuse scleroderma. Arthritis Rheum 43: 24372444, 2000.

4. Kahan A, Coghlan G, McLaughlin V. Cardiac complications of systemic sclerosis. Rheumatology (Oxford) 48 (Suppl 3): iii45-iii 
48, 2009.

5. Hachulla AL, Launay D, Gaxotte V, et al. Cardiac magnetic resonance imaging in systemic sclerosis: a cross-sectional observational study of 52 patients. Ann Rheum Dis 68: 1878-1884, 2009.

6. Corrado D, Fontaine G, Marcus FI, et al. Arrhythmogenic right ventricular dysplasia/cardiomyopathy: need for an international registry. Study Group on Arrhythmogenic Right Ventricular Dysplasia/Cardiomyopathy of the Working Groups on Myocardial and Pericardial Disease and Arrhythmias of the European Society of Cardiology and of the Scientific Council on Cardiomyopathies of the World Heart Federation. Circulation 101: E101-E106, 2000.

7. Sen-Chowdhry S, Lowe MD, Sporton SC, McKenna WJ. Arrhyth- mogenic right ventricular cardiomyopathy: clinical presentation, diagnosis, and management. Am J Med 117: 685-695, 2004.

8. Arai S, Kurasawa K, Maezawa R, Hanaoka R, Fukuda T. Systemic sclerosis complicated by arrhythmogenic right ventricular dysplasia that was misinterpreted as pulmonary arterial hypertension. Mod Rheumatol 22: 152-157, 2012.

9. Giusti B, Margheri F, Rossi L, et al. Desmoglein-2-integrin Beta-8 interaction regulates actin assembly in endothelial cells: deregulation in systemic sclerosis. PLoS One 8: e68117, 2013.

10. Jain A, Tandri H, Calkins H, Bluemke DA. Role of cardiovascular magnetic resonance imaging in arrhythmogenic right ventricular dysplasia. J Cardiovasc Magn Reson 10: 32, 2008.

(C) 2014 The Japanese Society of Internal Medicine http://www.naika.or.jp/imonline/index.html 